\title{
DISCOVERY OF A SUBSTELLAR COMPANION TO THE K2 III GIANT $\iota$ DRACONIS ${ }^{1}$
}

\author{
Sabine Frink, David S. Mitchell, and Andreas Quirrenbach \\ Center for Astrophysics and Space Sciences, University of California, San Diego, 9500 Gilman Drive, La Jolla, CA 92093-0424; \\ sabine@ucsd.edu,dsm@physics.ucsd.edu, aquirrenbach@ucsd.edu \\ Debra A. Fischer and Geoffrey W. Marcy \\ Department of Astronomy, University of California, Berkeley, 601 Campbell Hall, Berkeley, CA 94720-3411; \\ fischer@serpens.berkeley.edu,gmarcy@etoile.berkeley.edu \\ AND \\ R. PAul Butler \\ Department of Terrestrial Magnetism, Carnegie Institution of Washington, 5241 Broad Branch Road NW, Washington, DC 20015-1305; \\ paul@dtm.ciw.edu \\ Received 2002 March 21; accepted 2002 May 8
}

\begin{abstract}
We report precise radial velocity measurements of the K giant $\iota$ Dra (HD 137759, HR 5744, HIP 75458), carried out at Lick Observatory, which reveal the presence of a substellar companion orbiting the primary star. A Keplerian fit to the data yields an orbital period of about 536 days and an eccentricity of 0.70 . Assuming a mass of $1.05 M_{\odot}$ for $\iota$ Dra, the mass function implies a minimum companion mass $m_{2} \sin i$ of $8.9 M_{\mathrm{J}}$, making it a planet candidate. The corresponding semimajor axis is $1.3 \mathrm{AU}$. The nondetection of the orbital motion by Hipparcos allows us to place an upper limit of $45 M_{\mathrm{J}}$ on the companion mass, establishing the substellar nature of the object. We estimate that transits in this system could occur already for inclinations as low as 81.5 , as a result of the large diameter of the giant star. The companion to $\iota$ Dra is the first brown dwarf or planet found to orbit a giant rather than a main-sequence star.
\end{abstract}

Subject headings: astrometry — planetary systems — stars: individual ( $\iota$ Draconis)

On-line material: color figures

\section{INTRODUCTION}

Starting with the first detection of an extrasolar planet orbiting 51 Peg by Mayor \& Queloz (1995), numerous extrasolar planets and planet candidates have been and continue to be found via precise radial velocity searches (see, e.g., Marcy \& Butler 2000; Queloz et al. 2001). Many of the first candidates had short orbital periods and relatively high minimum masses corresponding to several Jupiter masses, reflecting the fact that those types of systems produce larger Doppler shifts and are thus easier to detect with the radial velocity technique.

The astrometric technique, which uses the shift in the position of the photocenter of the system imposed by the gravitational pull of the companion, is more sensitive to companions that are farther out, and the detection of longer periods is usually only limited by the duration of the respective observing program, usually a space mission. The accuracy of Hipparcos was not sufficient to detect extrasolar planets, although the signal of a few brown dwarf candidates seems to be present in the Hipparcos data (Halbwachs et al. 2000). Future astrometric space missions like $D I V A^{2}$ (Bastian et al. 1996), SIM $^{3}$ (see, e.g., NASA 1999; Unwin 1999; Fischer et al. 2001), and $G A I A^{4}$ (Perryman et al. 2001) are expected to dramatically change this situation by improving the astrometric accuracy by several orders of magnitude.

\footnotetext{
${ }^{1}$ Based on observations obtained at Lick Observatory, which is operated by the University of California.

${ }^{2}$ See http://www.ari.uni-heidelberg.de/diva.

${ }^{3}$ See http://sim.jpl.nasa.gov.

${ }^{4}$ See http://astro.estec.esa.nl/GAIA.
}

Other techniques that are capable of indirectly detecting the presence of an extrasolar planet include photometric searches for transiting planets (e.g., the Kepler ${ }^{5}$ or $\mathrm{COROT}^{6}$ missions) or the search for photometric or astrometric signatures of planetary companions in gravitational microlensing events.

Almost all extrasolar planet search programs that are underway already or planned to be launched in the future focus on late-type dwarf stars as host stars, the reasons for which are manifold. Since astronomers always strive to maximize the odds for finding what they are looking for, searching for extrasolar planets around solar-like stars is the natural first choice given the fact that we know that the Sun harbors nine planets. Furthermore, because of less severe rotational line broadening, precise radial velocities are much easier to measure for late-type stars than for earlytype ones, and in contrast to giants, dwarfs are known to display much smaller intrinsic radial velocity variations that could potentially conceal the signal of an extrasolar planet. Similarly, photometric searches are also far easier to carry out on small and dim stars such as late-type dwarfs as opposed to early-type or giant stars. And finally, late-type dwarfs are numerous, so that there is no shortage of wellsuited targets.

Since 1999 June our group has been monitoring the radial velocities of a sample of $\mathrm{K}$ giants at Lick Observatory with a precision of about $5-8 \mathrm{~m} \mathrm{~s}^{-1}$, only slightly less accurate than what is routinely achieved by the planet searches. The objective of this ongoing observing program is the statistical

\footnotetext{
${ }^{5}$ See http://www.kepler.arc.nasa.gov.

${ }^{6}$ See http://corot.astrsp-mrs.fr.
} 
study of the intrinsic radial velocity variability of $\mathrm{K}$ giants as preparation for SIM (see Frink et al. 2001). Thousands of astrometrically stable and thus single $\mathrm{K}$ giants are needed by SIM to provide an accurate astrometric reference frame, both locally and globally. The binary stars among a large K giant candidate sample of grid and reference stars have to be sorted out by means of a radial velocity survey before the mission. Our intention was to demonstrate the feasibility and to define the design parameters of this survey.

So while the detection of extrasolar planets or brown dwarfs was not the main motivation for carrying out this observing program, it was certainly within reach given our measurement accuracy and observing strategy. Here we report the first detection of a substellar companion orbiting a giant star rather than a dwarf. The mass of the companion makes it either a brown dwarf or a giant planet.

The outline of the paper is as follows. In $\S 2$ we describe our observations in more detail, followed by the derivation of the spectroscopic orbit in $\S 3$. In $\S 4$ we use the nondetection of the companion by Hipparcos to derive an upper limit for the mass of the companion, and $\S 5$ takes a closer look at the parent star. We discuss the possibility of transits in $\S 6$ and conclude with a short summary in $\S 7$.

\section{OBSERVATIONS}

The radial velocity observations were carried out with the $0.6 \mathrm{~m}$ coudé auxiliary telescope (CAT) and the attached Hamilton Echelle Spectrograph at Lick Observatory. An iodine cell was placed in the light path, and the resulting spectra with starlight and imprinted iodine absorption lines were fitted by superposition of an iodine template observation and a stellar template taken without the iodine cell. This technique is known to yield Doppler shifts accurate to $3 \mathrm{~m} \mathrm{~s}^{-1}$ and better for dwarf stars (Butler et al. 1996).

The resolution of the spectra is $R=60,000$ at $\lambda=6000$ $\AA$. The spectra cover the wavelength range from 4725 to $9590 \AA$ A before 2001 August and from 3755 to $9590 \AA$ after that. For the measurement of the radial velocities only the region from about 5000 to $5800 \AA$ is used, where most of the iodine lines can be found.

Typical exposure times for $\iota$ Dra (3.3 mag) were 5 minutes to achieve a signal-to-noise ratio of about 150, which yielded radial velocities with a precision of better than $5 \mathrm{~m}$ $\mathrm{s}^{-1}$. The individual radial velocities are listed in Table 1, along with Julian dates and derived formal errors. The zero level of the radial velocities was determined from the orbital fit described in the next section; the measurements themselves only provide relative radial velocities with an arbitrary zero point.

\section{THE SPECTROSCOPIC ORBIT}

Our measured radial velocities for $\iota$ Dra are shown in Figure 1, along with a Keplerian fit to the data. The orbit is very eccentric $(e=0.70)$, which is favorable for establishing a substellar companion as the reason for the observed radial velocity changes as opposed to stellar pulsations or rotational modulation of starspots. Furthermore, the reduced $\chi^{2}$ of the orbital fit is 8.5 , indicating that some additional

TABLE 1

Measured Radial Velocities for $\iota$ Dra

\begin{tabular}{|c|c|c|c|c|c|}
\hline $\begin{array}{c}\mathrm{JD}-\mathrm{JD}_{2000}{ }^{\mathrm{a}} \\
\text { (days) }\end{array}$ & $\begin{array}{c}v_{\mathrm{rad}} \\
\left(\mathrm{m} \mathrm{s}^{-1}\right)\end{array}$ & $\begin{array}{c}\sigma_{v_{\mathrm{rad}}} \\
\left(\mathrm{m} \mathrm{s}^{-1}\right)\end{array}$ & $\begin{array}{c}\mathrm{JD}-\mathrm{JD}_{2000}{ }^{\mathrm{a}} \\
\text { (days) }\end{array}$ & $\begin{array}{c}v_{\text {rad }} \\
\left(\mathrm{m} \mathrm{s}^{-1}\right)\end{array}$ & $\begin{aligned} \sigma_{v_{\text {rad }}} \\
\left(\mathrm{m} \mathrm{s}^{-1}\right)\end{aligned}$ \\
\hline $140.854 \ldots \ldots \ldots \ldots$ & -50.7 & 4.1 & $535.757 \ldots \ldots \ldots \ldots$ & -243.1 & 4.4 \\
\hline $162.754 \ldots \ldots \ldots \ldots$ & -19.2 & 10.1 & $536.766 \ldots \ldots \ldots \ldots$ & -215.1 & 4.3 \\
\hline $196.773 \ldots \ldots \ldots . .$. & 17.3 & 5.3 & $537.738 \ldots \ldots \ldots \ldots$ & -213.4 & 4.0 \\
\hline $237.678 \ldots \ldots \ldots \ldots$ & 56.5 & 4.6 & $538.721 \ldots \ldots \ldots \ldots$ & -213.6 & 3.5 \\
\hline $385.075 \ldots \ldots \ldots \ldots$ & 174.6 & 5.8 & $553.728 \ldots \ldots \ldots \ldots$ & -196.3 & 4.0 \\
\hline $404.082 \ldots \ldots \ldots \ldots$ & 204.2 & 6.3 & $554.707 \ldots \ldots \ldots \ldots$ & -188.0 & 4.1 \\
\hline $445.990 \ldots \ldots \ldots \ldots$ & 272.6 & 6.2 & $555.711 \ldots \ldots \ldots \ldots$ & -192.4 & 4.0 \\
\hline $466.948 \ldots \ldots \ldots \ldots$ & 58.6 & 4.9 & $561.718 \ldots \ldots \ldots \ldots$ & -193.3 & 5.3 \\
\hline $467.843 \ldots \ldots \ldots \ldots$ & 47.2 & 5.1 & $562.732 \ldots \ldots \ldots \ldots$ & -199.5 & 4.3 \\
\hline $468.010 \ldots \ldots \ldots \ldots$ & 30.8 & 4.6 & $563.702 \ldots \ldots \ldots \ldots$ & -193.8 & 4.1 \\
\hline $468.871 \ldots \ldots \ldots . .$. & 8.9 & 4.5 & $564.715 \ldots \ldots \ldots \ldots$ & -199.1 & 3.9 \\
\hline $468.996 \ldots \ldots \ldots \ldots$ & 0.4 & 5.7 & $565.700 \ldots \ldots \ldots \ldots$ & -183.7 & 4.1 \\
\hline $469.802 \ldots \ldots \ldots \ldots$ & -30.8 & 4.6 & $579.706 \ldots \ldots \ldots \ldots$ & -147.0 & 4.1 \\
\hline $469.997 \ldots \ldots \ldots . .$. & -44.9 & 5.0 & $580.708 \ldots \ldots \ldots \ldots$ & -164.5 & 4.6 \\
\hline $470.842 \ldots \ldots \ldots . .$. & -69.1 & 4.5 & $603.727 \ldots \ldots \ldots \ldots$ & -102.7 & 3.8 \\
\hline $470.991 \ldots \ldots \ldots . .$. & -41.9 & 4.4 & $618.638 \ldots \ldots \ldots \ldots$ & -111.8 & 5.0 \\
\hline $487.729 \ldots \ldots \ldots . .$. & -307.6 & 4.3 & $619.632 \ldots \ldots \ldots \ldots$ & -108.0 & 5.0 \\
\hline $487.975 \ldots \ldots \ldots \ldots$ & -321.1 & 4.8 & $620.624 \ldots \ldots \ldots \ldots$ & -110.4 & 4.8 \\
\hline $499.752 \ldots \ldots \ldots . .$. & -310.7 & 5.0 & $621.624 \ldots \ldots \ldots \ldots$ & -106.7 & 4.7 \\
\hline $499.926 \ldots \ldots \ldots \ldots$ & -312.8 & 4.4 & $629.608 \ldots \ldots \ldots \ldots$ & -101.9 & 5.1 \\
\hline $500.862 \ldots \ldots \ldots . .$. & -300.5 & 4.4 & $714.107 \ldots \ldots \ldots \ldots$ & -1.7 & 5.8 \\
\hline $501.803 \ldots \ldots \ldots . . .$. & -295.9 & 4.4 & $751.022 \ldots \ldots \ldots \ldots$ & 40.9 & 5.4 \\
\hline $501.926 \ldots \ldots \ldots \ldots$ & -310.9 & 4.9 & $753.079 \ldots \ldots \ldots \ldots$ & 28.7 & 6.5 \\
\hline $502.779 \ldots \ldots \ldots . . .$. & -293.6 & 4.3 & $763.017 \ldots \ldots \ldots \ldots$ & 72.1 & 5.9 \\
\hline $502.910 \ldots \ldots \ldots . .$. & -299.6 & 4.7 & $791.966 \ldots \ldots \ldots \ldots$ & 76.0 & 4.9 \\
\hline $503.741 \ldots \ldots \ldots \ldots$ & -282.9 & 4.5 & $816.971 \ldots \ldots \ldots \ldots$ & 59.1 & 5.5 \\
\hline $503.939 \ldots \ldots \ldots \ldots$ & -284.6 & 4.5 & $817.854 \ldots \ldots \ldots \ldots$ & 68.6 & 5.7 \\
\hline $534.759 \ldots \ldots \ldots . . .$. & -221.0 & 4.8 & $818.931 \ldots \ldots \ldots \ldots$ & 66.6 & 5.5 \\
\hline
\end{tabular}

${ }^{\mathrm{a}} \mathrm{JD}_{2000}=2,451,545.0$. 


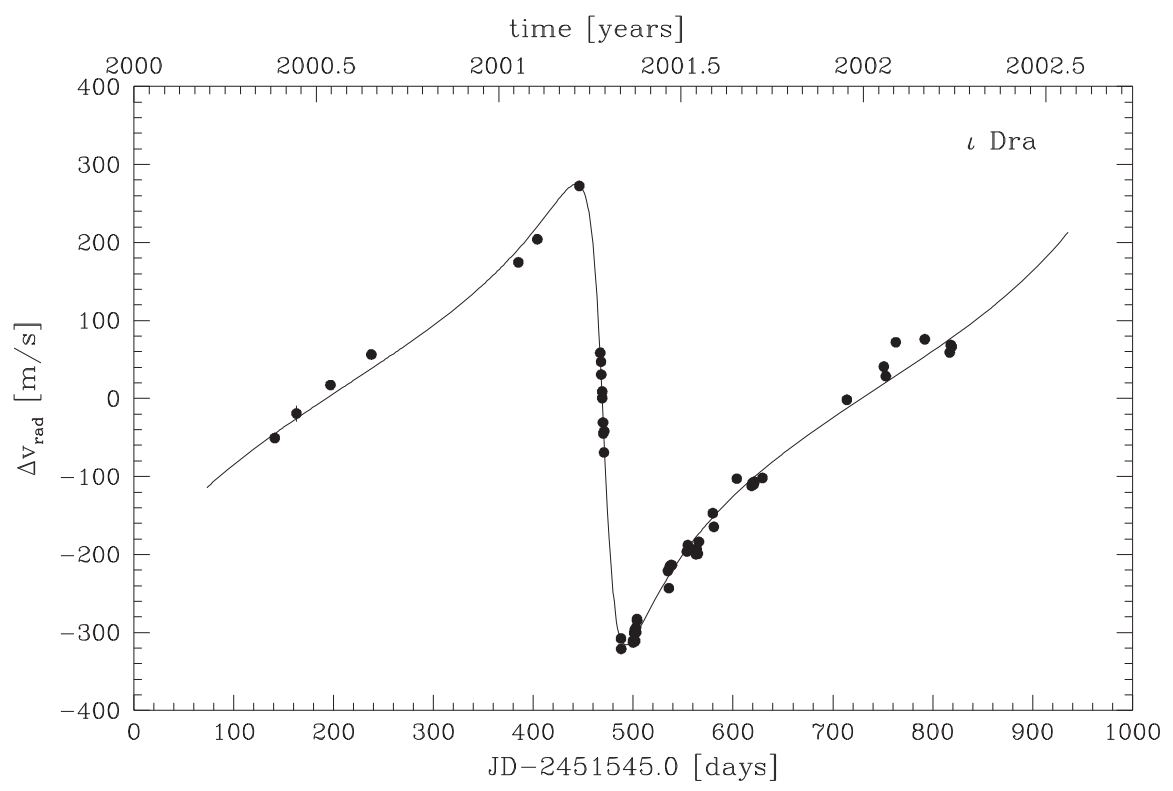

Fig. 1.-Measured relative radial velocities $\Delta v_{\text {rad }}$ of $\iota$ Dra over a period of about $2 \mathrm{yr}$. The measurement errors of the radial velocities are typically smaller than the symbol sizes, about $5 \mathrm{~m} \mathrm{~s}^{-1}$, but in a few cases the error bars are visible. Overplotted is the best-fit Keplerian orbit with a semiamplitude of $301.0 \mathrm{~m}$ $\mathrm{s}^{-1}$. [See the electronic edition of the Journal for a color version of this figure.]

radial velocity scatter of the order of $10 \mathrm{~m} \mathrm{~s}^{-1}$ is present. This sort of radial velocity scatter is typical for other K2 III giants in our sample, whereas a scatter of $150 \mathrm{~m} \mathrm{~s}^{-1}$ would clearly stand out (see Fig. 5 in Frink et al. 2001), indicating again an origin not intrinsic to the $\mathrm{K} 2$ giant itself as source for the observed radial velocity jitter.

There are six free parameters in the fit: period $P$, periastron time $T_{0}$, longitude of periastron $\omega$, eccentricity $e$, mass function, and the zero level of the radial velocities mentioned before. The fitted spectroscopic elements are listed in Table 2, along with estimated uncertainties based on the $\chi^{2}$ fit. The orbital elements are all very well determined, except for the period that is still uncertain to about 5 days. We will continue to observe $\iota$ Dra and expect to get a better constraint on the period once we have observed the second maximum in late August/early September of 2002; so far our observations just cover little more than one full $1.5 \mathrm{yr}$ period. A better knowledge of the period is important for possible transit detections; see the discussion in $\S 6$.

Unfortunately, no direct companion mass determination is possible from the spectroscopic orbit for those cases in which only one component is seen. For late-type main-

TABLE 2

Spectroscopic Orbital Elements for $\iota$ Dra

\begin{tabular}{|c|c|c|}
\hline Element & $\begin{array}{l}\text { Fitted } \\
\text { Value }\end{array}$ & $\begin{array}{l}\text { Estimated } \\
\text { Uncertainty }\end{array}$ \\
\hline Period $P$ (days)... & 536 & 5 \\
\hline Periastron time $T_{0} \ldots \ldots \ldots \ldots \ldots \ldots \ldots \ldots \ldots \ldots$ & $2,452,015.8^{\mathrm{a}}$ & $0.2^{\mathrm{b}}$ \\
\hline Longitude of periastron $\omega(\mathrm{deg}) \ldots \ldots \ldots .$. & 95.9 & 0.1 \\
\hline Eccentricity $e$ & 0.70 & 0.01 \\
\hline Mass function $f(m)^{\mathrm{c}}\left(M_{\odot}\right) \ldots \ldots \ldots \ldots \ldots$ & $5.1 \times 10^{-7}$ & $0.2 \times 10^{-7}$ \\
\hline
\end{tabular}

\footnotetext{
a JD.

${ }^{\mathrm{b}}$ In days.

c $f(m)=\left(m_{2} \sin i\right)^{3} /(m 1+m 2)^{2}$.
}

sequence primaries this is not a big concern, since masses are quite accurately known for these stars. However, giant masses are considerably less accurate. The best mass estimate for $\iota$ Dra available is $1.05 M_{\odot}$, which was derived by Allende Prieto \& Lambert (1999) based on Hipparcos observations (but see remarks in $\S 5$ ). This yields a minimum companion mass $m_{2} \sin i$ of $8.9 M_{\mathrm{J}}$.

From the fitted period and the assumed primary mass we derive a semimajor axis of 1.3 AU. However, the actual distance of the planet from the center of mass of the system varies between $0.4 \mathrm{AU}$ at periastron and 2.2 AU at apastron. For comparison, the radius of $\iota$ Dra is about $0.06 \mathrm{AU}$, so that the separation between the surface of $\iota$ Dra and its substellar companion at periastron is still at least $0.3 \mathrm{AU}$.

The inclination cannot be derived from radial velocity data, but in the next section we will derive a lower limit from the nondetection of orbital motion in the $\iota$ Dra system by Hipparcos.

\section{CONSTRAINTS FROM HIPPARCOS}

If the inclination of the orbital plane in the $\iota$ Dra system was very small (below about $6^{\circ}$ ) and thus the mass of the companion was close to the hydrogen-burning limit, the astrometric signature would have been large enough for Hipparcos to easily detect it (around 7 mas peak to peak). The Hipparcos intermediate astrometric data allow one to reexamine the individual Hipparcos abscissa measurements together with new constraints that come from different types of measurements. Sometimes it helps to detect an orbit in the data if one knows, e.g., period or eccentricity from radial velocity measurements, since this reduces the number of free parameters in the orbital fit.

This approach has been followed by Halbwachs et al. (2000), who reanalyzed the Hipparcos intermediate astrometric data for 11 spectroscopic binaries with possible brown dwarf secondaries, rejecting the brown dwarf 


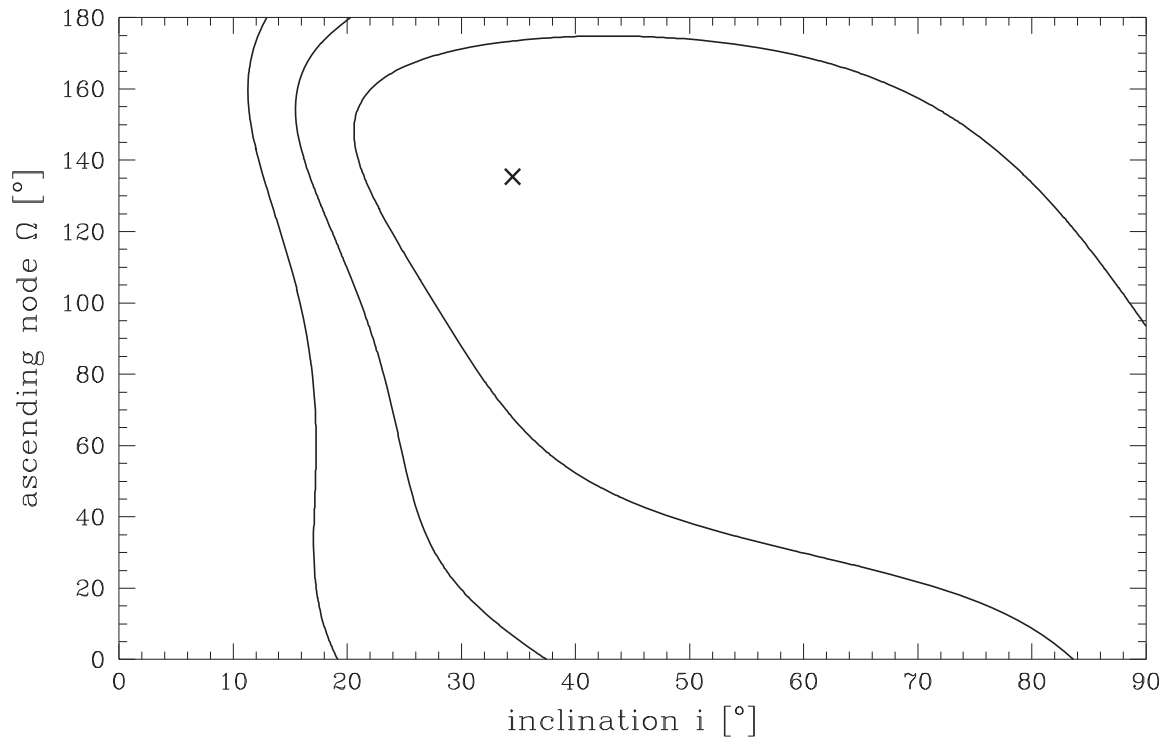

Fig. 2.- Contour plot for a $\chi^{2}$ fit to the Hipparcos intermediate astrometric data. The inclination $i$ and the ascending node $\Omega$ were the only two free parameters in the fit. The best-fit value ( $i=38^{\circ} .7, \Omega=126^{\circ} 3$ ) is indicated by a cross, and the contours represent the 1,2 , and $3 \sigma \chi^{2}$ values, respectively. The orbit is not detected in the Hipparcos data, but small values for the inclination can be rejected with high confidence. [See the electronic edition of the Journal for a color version of this figure.]

hypothesis for seven of those systems. Similarly, Mazeh et al. (1999) and Zucker \& Mazeh (2001) examined the Hippar$\cos$ data for 47 candidate planetary systems known from radial velocity searches as well as 14 brown dwarf candidates. They could confirm the substellar nature of 14 planetary companion candidates by deriving upper mass limits in the brown dwarf regime, without a real astrometric orbit detection, and confirmed the earlier results by Halbwachs et al. (2000) for brown dwarf candidates. Pourbaix (2001) and Pourbaix \& Arenou (2001) show that the Hipparcos data are in general not precise enough to derive astrometric orbits for substellar companions. However, the nondetection of an orbit by Hipparcos can still be used to derive an upper mass limit for an unseen companion.

The Hipparcos intermediate astrometric data archive provides 80 individual abscissa measurements for $\iota$ Dra (HIP 75458). They correspond to 41 different spacecraft orbits; for 39 orbits both consortia (FAST and NDAC) produced results, while for two orbits only one of the consortia came up with a solution. All abscissa measurements were properly decorrelated and weighted following the procedure described in van Leeuwen \& Evans (1998).

We then fitted an orbital solution to the data, leaving the orbital parameters from the radial velocity fit (Table 2 ) as well as the five astrometric parameters (positions, proper motions, and parallax) unchanged; the only two free parameters were inclination and ascending node.

Figure 2 shows the $\chi^{2}$ contours as a function of those two parameters. It is evident that the orbit is not detected in the Hipparcos data; the $3 \sigma$ contours almost span the entire parameter range, with the exception of the low inclination range. The reason why the fit can exclude low inclinations even if the orbit is not detected is because for low inclinations the astrometric signature would become larger than the abscissa residuals.

Based on our fit we can reject inclinations smaller than 11.3 at the $99.73 \%$ confidence level $(3 \sigma)$, which places an upper limit of $45 M_{\mathrm{J}}$ on the companion mass, well below the hydrogen-burning limit. With a probability of $68.3 \%$ the inclination is larger than $20 \%$, corresponding to a maximum mass of $25 M_{\mathrm{J}}$. Note that these probabilities are the formal ones usually quoted for $\chi^{2}$ fits. They do not take into account the distribution of inclinations according to $f(i) d i=\sin i d i$, nor the unknown mass function in that region. Low inclinations are extremely unlikely, and even without Hipparcos data the probability that the inclination was larger than 20.6 would have been $94 \%$ from statistical considerations.

We also tried to fit for the five astrometric parameters in conjunction with the orbit, but the differences between the published and fitted astrometric parameters were negligible. We also tried to keep the proper motion fixed at the slightly more accurate FK6 value instead of using the Hipparcos proper motion and fitted for the remaining three astrometric and two orbital parameters, but this did not result in changes to the fitted solution either.

Figure 3 shows the Hipparcos abscissa residuals along with the formal best-fit orbit of the $\iota$ Dra system with an inclination of $38^{\circ} .7$, corresponding to a companion mass of 14.2 $M_{\mathrm{J}}$. For illustration, the orbit for a companion with a mass of $0.08 M_{\odot}$, corresponding to an inclination of 6.1 , is also shown. The astrometric signature is clearly larger than the median abscissa error of 2.4 mas. The effects of parallax and proper motion have been removed in the plot; note also that abscissa values and model look close sometimes, but in general they do not correspond to the same time. The abscissa measurements are shown as circles only, although the measurements are onedimensional and are thus better represented by lines in the plot. However, that would make the illustration too crowded, and so only the position on this line with the shortest connection to the model position (without orbit) is shown. In other words, the measured position of $\iota$ Dra could be anywhere on a line perpendicular to the line 


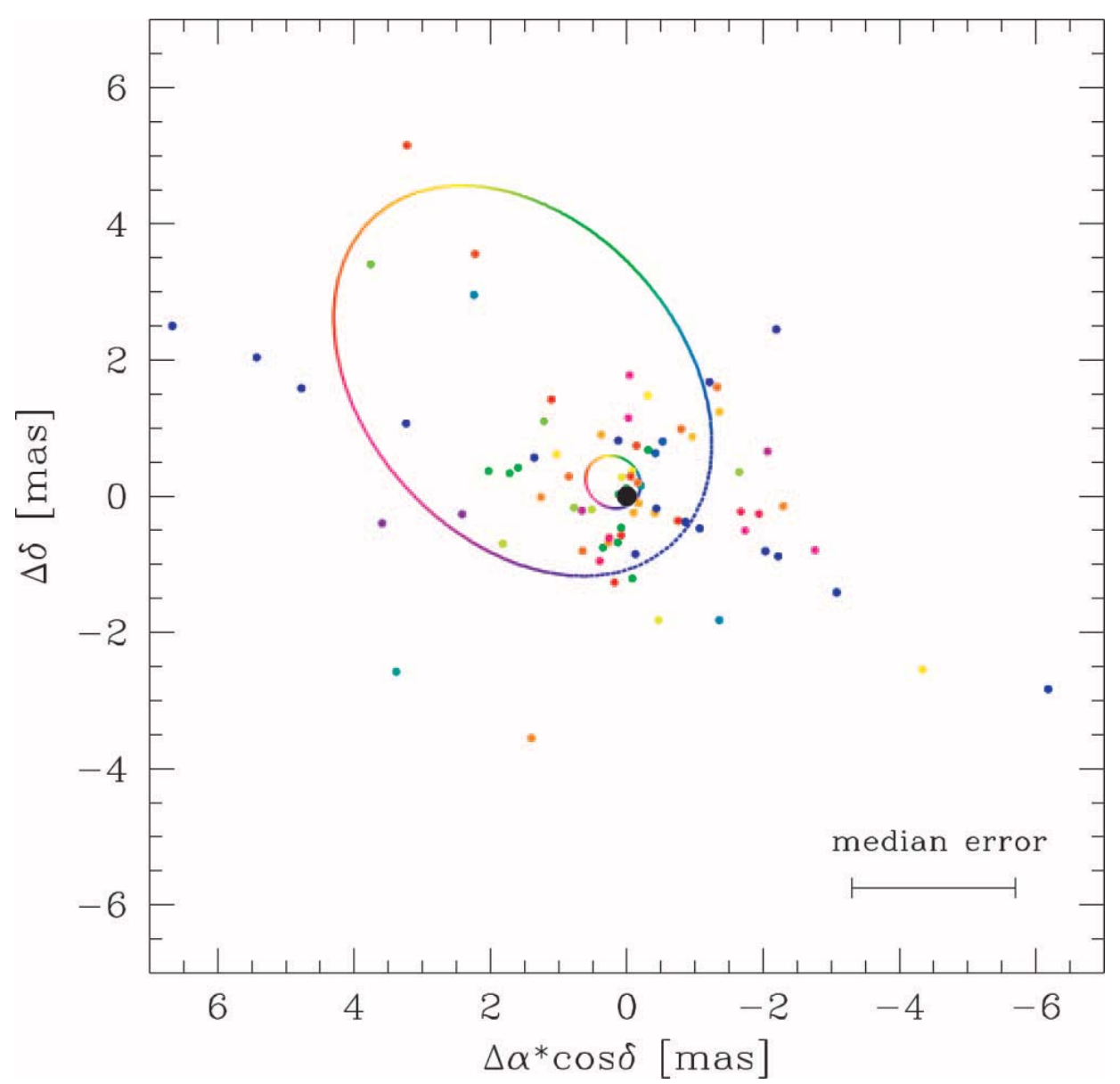

Fig. 3.-Illustration of the photocenter motion of $\iota$ Dra in the plane of the sky. The effects of proper motion and parallax have been removed. The position of $\iota$ Dra in the absence of orbital motion is indicated by the large central filled circle. The smaller ellipse illustrates the best-fit solution from Fig. 2, with an orbital inclination of 38.7 and a companion mass of $14.2 M_{\mathrm{J}}$. The larger ellipse represents the photocenter motion for the hypothetical case of a companion mass of $0.08 M_{\odot}$ (corresponding to an inclination of 6.1). The small filled circles represent the abscissa residuals for the case without orbital motion. The color represents orbital phase; abscissa measurements of a certain color correspond to the stretch on the ellipses of the same color. The actual abscissa measurements are only one-dimensional and would be represented by lines perpendicular to the directions connecting the individual small circles with the large central circle. The bar in the lower right shows the median standard error of 2.4 mas for the shown abscissa measurements. The data from both Hipparcos data reduction consortia, FAST and NDAC, are plotted.

passing through the large central circle and any given circle representing an abscissa measurement.

\section{THE HOST STAR ८ Dra}

\section{1. ८ Dra: A Visual Binary?}

The host star $\iota$ Dra is listed in the Washington Double Star (WDS; Worley \& Douglass 1997) catalog as a visual binary with a separation of 254".6 and a position angle of $50^{\circ}$. The observation dates back to 1879 . The fainter component is $\mathrm{BD}+59^{\circ} 1655\left(\iota\right.$ Dra itself is $\left.\mathrm{BD}+59^{\circ} 1654\right)$ with a $V$ magnitude of 8.76 mag in Tycho-1 (suspected to be intrinsically variable); at a distance of $31 \mathrm{pc}$ this would be compatible with a late $\mathrm{K}$ dwarf, and most catalogs list the spectral type as K7. Bartaya (1983), on the other hand, classified the potential secondary as an $\mathrm{M}$ giant, which would clearly place it at a much larger distance.

The system was considered a visual double on the basis of similar proper motions by Burnham. Consequently, it is also included in the Catalogue of the Components of Double and Multiple Stars (Dommanget \& Nys 1994) as CCDM $15250+5859$.
However, modern proper motions (see Table 3) show that

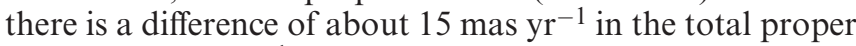
motion (or $2 \mathrm{~km} \mathrm{~s}^{-1}$ at a distance of $31 \mathrm{pc}$ ), which challenges the physical visual binary hypothesis. At a separation of 4.2 (confirmed by the modern positions), which corresponds to a physical separation of $7900 \mathrm{AU}$, the expected period is of the order of 500,000 days. For a circular orbit, this would correspond to a relative velocity of about $500 \mathrm{~m} \mathrm{~s}^{-1}$ or 3 mas $\mathrm{yr}^{-1}$, too small to explain the large observed propermotion differences. Significant eccentricity may be able to produce the observed difference in motions. However, we conclude that the evidence for a physical relation between the two stars is weak.

\subsection{Stellar Parameters of ८ Dra}

The mass of $\iota$ Dra has been determined to be $1.05 \pm 0.36$ $M_{\odot}$ by Allende Prieto \& Lambert (1999) via a comparison of theoretical isochrones by Bertelli et al. (1994) with the absolute visual magnitude and $B-V$ color based on Hipparcos data. Using the more recent evolutionary tracks by Yi et al. (2001) and their conversion to the observational $(B-V$, 
TABLE 3

Proper-Motion Comparison for $\iota$ Dra and Its Possible Visual Companion

\begin{tabular}{|c|c|c|c|c|c|c|c|c|c|c|}
\hline \multirow[b]{2}{*}{ Catalog } & \multicolumn{5}{|c|}{$\iota$ DRA } & \multicolumn{5}{|c|}{$\mathrm{BD}+59^{\circ} 1655$} \\
\hline & Number & $\begin{array}{c}\mu_{\alpha *} \\
\left(\operatorname{mas~yr}^{-1}\right)\end{array}$ & $\begin{array}{c}\mu_{\delta} \\
\left(\text { mas yr }^{-1}\right)\end{array}$ & $\begin{array}{c}\sigma_{\mu_{\alpha} *} \\
\left(\mathrm{mas} \mathrm{yr}^{-1}\right)\end{array}$ & $\begin{array}{c}\sigma_{\mu_{\delta}} \\
\left(\mathrm{mas} \mathrm{yr}^{-1}\right)\end{array}$ & Number & $\begin{array}{c}\mu_{\alpha *} \\
\left(\operatorname{mas~yr}^{-1}\right)\end{array}$ & $\begin{array}{c}\mu_{\delta} \\
\left(\text { mas yr }^{-1}\right)\end{array}$ & $\begin{array}{c}\sigma_{\mu_{\alpha} *} \\
\left(\operatorname{mas~yr}^{-1}\right)\end{array}$ & $\begin{array}{c}\sigma_{\mu_{\delta}} \\
\left(\operatorname{mas~yr}^{-1}\right)\end{array}$ \\
\hline FK6 ............. & 571 & -8.17 & 16.34 & 0.25 & 0.25 & $\ldots$ & $\ldots$ & $\ldots$ & $\ldots$ & $\ldots$ \\
\hline Hipparcos....... & 75458 & -8.27 & 17.30 & 0.38 & 0.49 & $\ldots$ & $\ldots$ & $\ldots$ & $\ldots$ & $\ldots$ \\
\hline PPM .............. & 34972 & -9 & 17. & 0.4 & 0.4 & 34976 & 3. & -8 & 3.3 & 3.5 \\
\hline ACT ................ & 38751620 & -12.1 & 6.2 & 3.47 & 4.88 & 3875232 & 1.7 & -6.9 & 1.66 & 2.17 \\
\hline TRC................. & 38751620 & -14.2 & 14.6 & 2.2 & 1.8 & 3875232 & 1.4 & -4.7 & 2.1 & 4.7 \\
\hline Tycho-2 .......... & 38751620 & -8.1 & 16.2 & 0.7 & 0.8 & 3875232 & 0.5 & -5.6 & 1.2 & 1.4 \\
\hline
\end{tabular}

$M_{V}$ )-plane yields a stellar mass that is perfectly consistent with the mass derived by Allende Prieto \& Lambert (1999).

This mass estimation seems to be the best one available today, and we have used it in calculating the companion mass. However, we caution that the tracks for different masses are quite close in this part of the HertzsprungRussell diagram, so that slight changes in the evolutionary models could have large effects on the derived masses. Giant masses are in general much more uncertain than those for main-sequence stars, and older evolutionary tracks seem to yield slightly higher masses.

Allende Prieto \& Lambert (1999) also derived the effective temperature $\log T_{\text {eff }}(\mathrm{K})=3.65 \pm 0.01$, the surface gravity $\log g\left(\mathrm{~cm} \mathrm{~s}^{-2}\right)=2.24 \pm 0.35$, and the radius $\log R\left(R_{\odot}\right)=$ $1.11 \pm 0.02$. This is in good agreement with other determinations (see Table 4). We also list the metallicities derived by various authors, most of which are compatible with almost solar metallicity, $[\mathrm{Fe} / \mathrm{H}]=0.03-0.06 \mathrm{dex}$, for $\iota$ Dra, while two authors derived somewhat higher values. A luminosity of $\log \left(L / L_{\odot}\right)=1.848 \pm 0.08$ was derived by Mallik (1999).

The stellar diameter has been estimated to be 6.0 and 4.9 mas by Hertzsprung (1922) and Wesselink, Paranya, \& de Vorkin (1972), respectively, which, at a distance of $31 \mathrm{pc}$, is only slightly larger than the newer radius determinations based on evolutionary models.

\section{PROSPECTS FOR TRANSITS}

The probability that the inclination of the system is large enough for transits to occur is larger than for planetary companions around main-sequence stars since the stellar disk of the giant is so much larger. Furthermore, the orbit geometry of the $\iota$ Dra system is favorable in so far as possible transits would occur close to periastron, when the separation between the two components is smallest, thus increasing again the range in inclinations for which transits can occur.

Based on the orbit geometry and the radius of $\iota$ Dra we estimate that transits could possibly occur for inclinations larger than $81^{\circ} .5$. The transit would last 3.5 days if the inclination was $90^{\circ}$ and somewhat shorter for smaller inclinations. Using the current orbital solution, we predict that the next transit for the $\iota$ Dra system would occur around 2002 October 3.

However, while the probability for transits to occur is larger for systems with a giant star, they are much more difficult to detect. While the dip in the photometric light curve of a main-sequence star resulting from the occultation of part of the stellar disk by a Jupiter-size planet is of the order of $1 \%$, this percentage is much smaller for a giant star system, about $0.01 \%$. For comparison, the photometric precision achieved in the transit detection of HD 209458 with the Hubble Space Telescope by Brown et al. (2001) was 0.011\%, about the size of the expected signal. The Kepler mission, scheduled to launch in 2006, aims for a photometric precision of $0.001 \%$, which would be sufficient to detect this kind of transit.

\section{SUMMARY}

We present precise radial velocity observations of the K2 III giant $\iota$ Dra that reveal the presence of a substellar object in orbit around the $\mathrm{K}$ giant. From a Keplerian fit to the data we determine a period of about 536 days and an eccentricity of 0.70 . Some additional scatter of the order of $10 \mathrm{~m} \mathrm{~s}^{-1}$ is present, which is typical for early $\mathrm{K}$ giants and very likely intrinsic to the star. Assuming a mass of $1.05 M_{\odot}$ for $\iota$ Dra, we derive a minimum companion mass $m_{2} \sin i$ of $8.9 M_{\mathrm{J}}$,

TABLE 4

Comparison of the Stellar Parameters for $\iota$ Dra Derived by Various Authors

\begin{tabular}{|c|c|c|c|c|c|c|}
\hline Reference & $\begin{array}{l}T_{\text {eff }} \\
(\mathrm{K})\end{array}$ & $\begin{array}{l}\sigma_{T_{\text {eff }}} \\
(\mathrm{K})\end{array}$ & $\begin{array}{c}\log g \\
\left(\mathrm{~cm} \mathrm{~s}^{-2}\right)\end{array}$ & $\begin{array}{c}\sigma_{\log g} \\
\left(\mathrm{~cm} \mathrm{~s}^{-2}\right)\end{array}$ & {$[\mathrm{Fe} / \mathrm{H}]$} & $\sigma_{[\mathrm{Fe} / \mathrm{H}]}$ \\
\hline Prugniel \& Soubiran 2001 ............... & 4491 & $\ldots$ & 2.57 & $\ldots$ & 0.06 & $\ldots$ \\
\hline Cenarro et al. 2001 ......................... & 4498 & $\ldots$ & 2.380 & $\ldots$ & 0.05 & $\ldots$ \\
\hline Allende Prieto \& Lambert 1999 ....... & 4466 & 100 & 2.24 & 0.35 & $\ldots$ & $\ldots$ \\
\hline 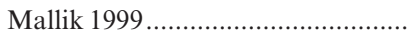 & 4553 & 50 & $\ldots$ & $\ldots$ & $\ldots$ & $\ldots$ \\
\hline 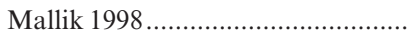 & 4400 & $\ldots$ & $\ldots$ & $\ldots$ & 0.33 & $\ldots$ \\
\hline McWilliam $1990 \ldots \ldots \ldots \ldots \ldots \ldots \ldots \ldots \ldots$ & 4490 & $\ldots$ & 2.74 & $\ldots$ & 0.03 & 0.11 \\
\hline Williams 1974 ............... & 4530 & 100 & 2.60 & 0.25 & 0.29 & 0.2 \\
\hline
\end{tabular}


which makes the companion a giant planet candidate and the first substellar object found orbiting a giant star.

Furthermore, we used the nondetection of the orbit by Hipparcos to reject inclinations smaller than 11.3 at the $3 \sigma$ level. The corresponding upper limit for the companion mass is $45 M_{\mathrm{J}}$, establishing the substellar nature of the object. Finally, we estimate that possible transits could occur already for inclinations as low as 81.5 , as a result of the large diameter of the giant star. An unambiguous measurement of the inclination, needed to distinguish between the planetary and brown dwarf nature of the companion, has to await the launch of astrometric satellites such as the planned DIVA, SIM, and GAIA missions.

It is a pleasure to thank the staff at Lick Observatory for their continuous support and assistance. S. F. and A. Q. gratefully acknowledge support from NASA's SIM Preparatory Science Program.
Allende Prieto, C. \& Lambert, D. L 1999, A\&A, 352, 555

Bartaya, R. A. 1983, Bull. Inf. CDS, 24, 93

Bastian, U., et al. 1996, Astron. Nachr., 317, 281

Bertelli, G., Bressan, A., Chiosi, C., Fagotto, F., \& Nasi, E. 1994, A\&AS, 106,275

Brown, T. M., Charbonneau, D., Gilliland, R. L., Noyes, R. W., \& Burrows, A. 2001, ApJ, 552, 699

Butler, R. P., Marcy, G. W., Williams, E., McCarthy, C., \& Dosanjh, P. 1996, PASP, 108, 500

Cenarro, A. J., Gorgas, J., Cardiel, N., Pedraz, S., Peletier, R. F., \& Vazdekis, A. 2001, MNRAS, 326, 981

Dommanget, J., \& Nys, O. 1994, Commun. Obs. R. Belgique, 115, 1

Fischer, D. A., Marcy, G. W., Oppenheimer, B. R., Quirrenbach, A., Frink, S., Monet, D. G., Scargle, J., \& Butler, R. P. 2001, AAS, 198, 62.08

Frink, S., Quirrenbach, A., Fischer, D., Röser, S., \& Schilbach, E. 2001, PASP, 113, 173

Halbwachs, J. L., Arenou, F., Mayor, M., Udry, S., \& Queloz, D. 2000, A\&A, 355, 581

Hertzsprung, E. 1922, Ann. Sternwarte Leiden XIV, 1

Mallik, S. V. 1998, A\&A, 338, 623

- 1999, A\&A, 352, 495

\section{REFERENCES}

Marcy, G. W., \& Butler, R. P. 2000, PASP, 112, 137

Mayor, M., \& Queloz, D. 1995, Nature, 378, 355

Mazeh, T., Zucker, S., dalla Torre, A., \& van Leeuwen, F. 1999, ApJ, 522 L149

McWilliam, A. 1990, ApJS, 74, 1075

NASA 1999, SIM Space Interferometry Mission: Taking the Measure of the Universe, ed. R. Danner \& S. Unwin, JPL 400-811

Perryman, M. A. C., et al. 2001, A\&A, 369, 339

Pourbaix, D. 2001, A\&A, 369, L22

Pourbaix, D., \& Arenou, F. 2001, A\&A, 372, 935

Prugniel, Ph., \& Soubiran, C. 2001, A\&A, 369, 1048

Queloz, D., et al. 2001, Messenger, 105, 1

Unwin, S. C. 1999, AAS, 31, 14.03

van Leeuwen, F., \& Evans, D. W. 1998, A\&AS, 130, 157

Wesselink, A. J., Paranya, K., \& de Vorkin, K. 1972, A\&AS, 7, 257

Williams, P. M. 1974, MNRAS, 167, 359

Worley, C. E., \& Douglass, G. G. 1997, A\&AS, 125, 523

Yi, S., Demarque, P., Kim, Y., Lee, Y., Ree, C. H., Lejeune, T., \& Barnes, S. 2001, ApJS, 136, 417

Zucker, S., \& Mazeh, T. 2001, ApJ, 562, 549 